\title{
Los pastores vascos y la guerra de las praderas. Inmigración vasca, industria ganadera y conflictos por las tierras federales en el estado de Nevada, 1890-1934 ${ }^{1}$
}

\author{
Iker Saitua ${ }^{2}$
}

Recibido: 21 de diciembre de 2016 / Aceptado: 16 de mayo de 2017

\begin{abstract}
Resumen. Este artículo analiza los conflictos en torno al pastoreo de tierras públicas en el estado de Nevada durante el primer tercio del siglo XX, que enfrentó a ganaderos bovinos y pastores trashumantes de ovejas, así como los intentos del gobierno federal por mitigarlos y poner orden en las praderas del Oeste. Se propone explicar cómo los ganaderos bovinos intentaron afianzar su posición económica sobre las explotaciones ganaderas más pequeñas, enfrentándose a los ovejeros, de posición social más débil y en su mayoría inmigrantes vascos.

Palabras clave: Inmigración vasca a Estados Unidos; Western History; Great Basin; Nevada; Range Wars; U.S. Forest Service; tierras públicas; ganadería extensiva; pastores de ovejas.
\end{abstract}

[en] Basque Sheepherders and the Range Wars. Basque Immigration, Livestock Industry, and the Conflicts over the Use of Federal Lands in the State of Nevada, 1890-1934

\begin{abstract}
This article analyzes the conflicts between cattle ranchers and sheepherders over the use of the public-domain lands during the first third of the twentieth century, as well as the federal government's attempts to alleviate these conflicts and bring order to the western rangelands. The article examines how cattle ranchers tried to secure their economic status over smaller operations, confronting sheepherders who belonged to a lower social-economic status and most of them were Basque immigrants.

Keywords: Basque Immigration in the United States; Western History; Great Basin; Nevada; Range Wars; U.S. Forest Service; Public Lands; Extensive Ranching; Sheepherders.
\end{abstract}

Sumario. Introducción. 1. La ganadería extensiva y sus problemas en Nevada a finales del siglo XIX. 2. Los pastores vascos y la guerra de las praderas. 3. La regularización del uso de la tierra de pastoreo en Nevada. El nuevo régimen de aprovechamiento forestal y el sector ganadero de Nevada. 4. El lobby ganadero de Nevada, el problema de las tierras públicas y los pastores vascos. 5. Conclusiones. 6. Referencias bibliográficas.

Cómo citar: Saitua, I. (2017). "Los pastores vascos y la guerra de las praderas. Inmigración vasca, industria ganadera y conflictos por las tierras federales en el estado de Nevada, 1890-1934". Cuadernos de Historia Contemporánea, 39, 191-211.

\footnotetext{
1 Universidad de Nevada-Reno (UNR), programa de doctorado.

2 Universidad del País Vasco UPV/EHU (España)

E-mail: saituaidarraga@gmail.com
} 


\section{Introducción ${ }^{3}$}

En diciembre de 1913, un periódico local del estado de Nevada anunciaba de la siguiente manera que las luchas entre ganaderos y agricultores por el libre aprovechamiento de las tierras federales dedicadas al pastoreo, que tanto habían marcado la historia contemporánea del Oeste norteamericano, habían concluido: "RANGE WARS ARE ENDED”. Según este periódico, el nuevo régimen legal sobre el aprovechamiento de los recursos forestales del país, bajo la administración del Servicio Forestal de los Estados Unidos (U.S. Forest Service) creado en 1905 con objeto de asegurar una gestión sostenible y la conservación de las tierras forestales de dominio público, había puesto fin a estas reyertas en Nevada para siempre:

Twenty million head of stock were grazed in the forest reserves of the country in 1913 , returning a revenue therefor of about $\$ 2.500 .000$. The government's policy in regard to public land grazing privileges has been instrumental in doing away with the range wars that once formed a part of western range life, picturesque, though anarchistic ${ }^{4}$.

A comienzos del siglo $\mathrm{XX}$, en medio de un panorama de incertidumbre tanto a nivel nacional como internacional, el gobierno federal estadounidense había llegado a la conclusión de que era necesaria una intervención gubernamental para proteger el medio ambiente y regular las industrias que habían explotado los recursos naturales del Oeste norteamericano. La creación en 1905 de una agencia gubernamental para la conservación de los bosques del país, el Forest Service, reveló la preocupación del gobierno central por el agotamiento y destrucción de los recursos naturales a causa de la vertiginosa industrialización del Oeste durante la segunda mitad del siglo XIX. Aunque este primer paso para la conservación del medioambiente abrió el camino para las posteriores medidas de gestión medioambiental en los Estados Unidos, tuvieron que pasar algunos años antes de que desapareciesen por completo los conflictos ganaderos relacionados con el uso de las tierras públicas y la gestión del agua en el estado de Nevada.

A comienzos del siglo XX, una serie de grandes compañías ganaderas de Nevada comenzaron a reclamar derechos de propiedad reales sobre las tierras públicas argumentando que habían contribuido a mejorar su valor económico durante años más que ningún otro tipo de ganaderos. A través de sus representantes en Washington D.C., y formando un lobby de presión, exigieron al gobierno federal un nuevo sistema de uso de la tierra que les permitiese asegurar una posición privilegiada y dominante frente a otras explotaciones ganaderas más pequeñas. Para los grandes ganaderos de Nevada y para el emergente movimiento conservacionista, el blanco habitual fueron los inmigrantes vascos que componían una gran parte de la mano de obra en las explotaciones de ganado ovino, trabajando como pastores nómadas ${ }^{5}$. El

3 Este artículo adelanta algunas cuestiones tratadas en mi tesis doctoral, defendida en la Universidad de NevadaReno (UNR) en abril de 2016 con el título Sagebrush Laborers: Basque Immigrants in Nevada's Sheep Industry, International Dimensions, and the Making of an Agricultural Workforce, 1880-1954.

4 Reno Evening Gazette, 26-XII-1913, p. 4.

5 No he podido cuantificar el número exacto de inmigrantes vascos en Nevada entre las fechas objeto de estudio puesto que los censos de inmigración no diferencian otra categoría que españoles o franceses (en las que están sumergidos los inmigrantes vascos). No obstante, a través de otras fuentes, tales como prensa periódica o 
sentimiento nativista de la época se convirtió en un enérgico instrumento en su batalla para mantener el monopolio de la tierra en esta región del país. Cuando estalló la Primera Guerra Mundial y la demanda de productos ganaderos aumentó considerablemente, este sentimiento, que rechazaba como foráneo todo lo que no encajase en sus parámetros de identificación, creció y sirvió de pretexto para que estos ganaderos reclamasen una protección gubernamental que les favoreciese frente a otras explotaciones. Los pastores inmigrantes vascos fueron percibidos entonces como una amenaza extranjera y por ello perseguidos, resultando altamente vulnerables ante las desenfrenadas luchas en las praderas que se desencadenaron. Estudiar este conflicto es el objetivo de las páginas que siguen.

\section{La ganadería extensiva y sus problemas en Nevada a finales del siglo XIX}

Durante la Guerra Civil estadounidense (1861-1865), el Oeste árido contempló un aumento considerable de la producción ganadera que se incrementó en los años posteriores. La expansión de las actividades pecuarias creció en las décadas de 1870 y 1880, tras la construcción del ferrocarril Transcontinental, que aceleró la población de las zonas más marginales del Oeste norteamericano, como la Gran Cuenca. Debido a las características ambientales de esta región (en especial, la escasez de agua) el desarrollo de la agricultura estaba muy limitado. Por eso, a finales del siglo XIX, la producción del ganado en forma extensiva era una de las pocas empresas que había prosperado en estas tierras ${ }^{6}$.

Durante años, los ganaderos de Nevada y del Oeste árido utilizaron las tierras públicas para dar de pastar a sus animales como mejor les placía. La escasez de forrajes para la alimentación del ganado en este estado significaba que los ganaderos tenían que desplazarse largas distancias en busca de alimento. Debido a las características ecológicas del Oeste árido y semiárido, para que una empresa ganadera fuese viable era necesario contar con grandes extensiones de tierra. Pero la tenencia de amplias superficies requería de grandes inversiones de capital, lo que aumentaba los gastos de producción. Por eso, en un estado como Nevada, lo que resultaba decisivo para sustentar una mayor cabaña ganadera era el uso extensivo de las tierras públicas, que eran propiedad del gobierno federal. Este sistema ganadero era conocido como Open Range, y constituye uno de los rasgos esenciales de la historia agraria del Oeste norteamericano de este periodo ${ }^{7}$.

La mano de obra contratada por las explotaciones agropecuarias para cuidar de sus animales, ya fueran los pastores de ovejas o sus homólogos en el sector vacuno que montaban a caballo -los cowboys-, deambulaba libremente por los pastos públi-

documentación epistolar, puede deducirse que eran numéricamente importantes y que eran un colectivo definido y relevante en el sector ovino. Para 1910 se calcula que el número de inmigrantes vascos en los estados de California, Nevada, Idaho y Wyoming fue aproximadamente de 8,400. Arrizabalaga, Marie Pierre: A Statistical Study of Basque Immigration into California, Nevada, Idaho and Wyoming Between 1900 and 1910, tesis de master, University of Nevada, Reno, 1986, pp. 53-54.

6 White, Richard: "It's Your Misfortune and None of My Own": A New History of the American West, Norman, University of Oklahoma Press, 1991, pp. 270-272.

7 Young, James A. y Sparks, B. Abbott: Cattle in the Cold Desert, Logan, Utah State University Press, 1985, pp. 48-56; Rowley, William: "From Open Range to Closed Range on the Public Lands", en William G. Robbins y James C. Foster (eds.): Land in the American West: Private Claims and the Common Good, Seattle-London, University of Washington Press, 2000, pp. 96-98. 
cos -que eran pobres y no habían sido reclamados por su escaso valor económico-, buscando las mejores tierras, de las que se beneficiaban un número restringido de personas. Los ganaderos utilizaban estas tierras del gobierno con su consentimiento y sin pagar ningún tributo a cambio. En ellas, como ha explicado el historiador Samuel Hays, predominó el caos y la anarquía. La inexistencia de los aparatos del Estado en su regulación permite entender las cruentas batallas que se dieron entre ganaderos por el acceso y utilización de estos pastos. Estas luchas, popularmente conocidas como Range Wars, se prolongaron hasta la década de 1930, siendo este periodo uno de los capítulos más sangrientos de la historia del Oeste de los Estados Unidos. Aunque muchos ganaderos (grandes y pequeños) murieron en estas reyertas, el mayor perjudicado fue, como escribió la historiadora E. Louise Peffer, las propias praderas $^{8}$.

Desde la década de 1860, la ganadería extensiva sobreexplotó la capacidad nutritiva del pasto debido al incremento del número de cabezas de animales año tras año. A esto hay que sumar el deterioro del suelo ocasionado por la labranza tradicional de los colonos agricultores que se habían asentado en Nevada y otros estados del Oeste árido, a los que el gobierno federal había concedido parcelas de tierras públicas con el propósito de mejorar la productividad de las mismas y al mismo tiempo estabilizar la situación socio-económica. Estas intensas roturaciones hicieron retroceder las tierras de pasto en Nevada, que ya de por sí eran marginales y de escaso rendimiento. Por lo general, estos colonos, cuyos intereses con frecuencia colisionaban con la mayoría de los productores ganaderos, no consiguieron prosperar con sus explotaciones agrícolas debido a las dificultades derivadas de la escasez de agua y la naturaleza del terreno; la mayoría de ellos fracasaron en el intento. Las roturaciones excesivas fomentadas por estas granjas agrícolas, junto con los duros inviernos de finales de 1880 y la expansión de la ganadería extensiva, aceleraron la erosión del suelo y el proceso de desertificación 9 .

En la década de 1890, existía una total incertidumbre y mucho riesgo en la ganadería de Nevada. La mayoría de los ganaderos pastaban en las tierras públicas, pero eran tierras deterioradas sobre las que tenían cada vez menos control. La expansión del sector ovino aumentó la frustración de los productores de carne vacuna, pues parte de estos pastos eran tierras y aguas adyacentes a sus propiedades privadas. El gobierno de Nevada, aunque a favor del sector vacuno, no tenía poder para ejecutar políticas relativas al aprovechamiento de recursos naturales sobre estas tierras de pasto, pues esta era una atribución federal, pero para poder hacer frente a las guerras de las praderas tuvo que acabar entrando a legislar en este asunto. Desde 1890 se venía asistiendo a una fuerte reducción de la productividad del suelo que obligó a acometer una importante transformación del sector ganadero en la región.

La modificación más importante que tuvo lugar en la ganadería durante la década de 1890 fue un aumento de la privatización de la tierra. Hasta entonces, las explotaciones ganaderas no se habían preocupado de acumular grandes excedentes ni reservas de pastos para hacer frente a los tiempos de escasez, ya que, dada la abundancia

8 Hays, Samuel P.: Conservation and the Gospel of Efficiency: The Progressive Conservation Movement, 18901920, Cambridge, Harvard University Press, 1959, pp. 49-51; Peffer, Louise E.: The Closing of the Public Domain: Disposal and Reservation Policies, 1900-1950, Stanford, Stanford University Press, 1951, p. 26.

9 Patterson, Edna B., Ulph, Louise A. y Goodwin, Victor: Nevada's Northeast Frontier, Sparks, Western Printing and Publishing Company, 1969, pp. 211-213. 
de tierras baldías, el pasto estaba garantizado. Sin embargo, desde 1890, a causa de la inestabilidad económica creada por el sobrepastoreo y una preocupante crisis ambiental, se hizo manifiesta la necesidad de comprar tierras para la producción de pasto cultivado, con el fin de proporcionar una mayor seguridad a las explotaciones agropecuarias frente a las frecuentes sequías y otras adversidades climáticas del Oeste árido. Así, desde entonces, un número significativo de ganaderos, que aspiraban a ser hacendados importantes en el Oeste, adquirieron terrenos, bien de manera fraudulenta o bien legítimamente mediante compra a terceros de títulos de propiedad sobre la tierra. Durante el primer tercio del siglo XX, este fenómeno aumentó, aunque la gran mayoría de ganaderos continuó utilizando las tierras federales como complemento a los pastos privados. La trashumancia ganadera no desapareció por completo, pero sí se fue produciendo un estrangulamiento progresivo de este sistema de pastoreo extensivo en Nevada ${ }^{10}$.

Hasta la década de 1890 la proporción de ganado lanar fue inferior a la de vacuno, entre otras cosas porque las ovejas eran menos populares. Varios desastres naturales consecutivos y en particular el devastador invierno de 1889-1890 sumergieron al sector ganadero en una crisis larga y profunda, una de cuyas consecuencias más destacables fue el descenso considerable del número de cabezas vacunas. Las explotaciones ovejeras sufrieron menos bajas, ya que la oveja doméstica se distingue de otras especies ganaderas por su capacidad para sobrevivir en regiones áridas y desérticas. A partir de este momento, el ganado ovino aumentó considerablemente en detrimento del vacuno debido a las ventajas de la producción lanar. La oposición entre ganaderos trashumantes y estantes se recrudeció en la década de 1890 con la llegada de nuevos migrantes que trataban de probar suerte en la ganadería extensiva de ovinos y de sacar provecho de las tierras públicas ${ }^{11}$.

\section{Los pastores vascos y la guerra de las praderas}

Entre 1880 y 1920, la mano de obra vasca se había convertido en una característica estructural de la industria ovina del Oeste norteamericano. A finales del siglo XIX, tras el boom que experimentó la ganadería ovina en el Oeste árido, muchos inmigrantes vascos, como otras colectividades inmigrantes del sur de Europa, encontraron trabajo como pastores de grandes rebaños de ovejas. Muchos de ellos habían emigrado del País Vasco por razones económicas. El flujo de vascos de zonas rurales hacia el Oeste norteamericano responde principalmente a tres factores: en primer lugar, la crisis socio-económica en el mundo rural durante los años de la consolidación de la sociedad capitalista moderna en el País Vasco; en segundo lugar, los profundos cambios estructurales que experimentan las provincias vascas en la segunda mitad del siglo XIX tras la abolición del régimen foral; y en tercer lugar, el sistema de primogenitura vasco por el cual el varón mayor de la familia heredaba las posesiones de la familia, quedando prácticamente excluido el resto de los hijos ${ }^{12}$.

10 Young y Sparks: Cattle..., pp. 130-151

11 Sawyer, Byrd W.: Nevada Nomads: A Story of the Sheep Industry, San Jose, Harlan-Young Press, 1971, pp. 7889.

12 Douglass, William A. y Bilbao, Jon: Amerikanuak: Basques in the New World, Reno, University of Nevada Press, 1975, pp. 123-137; Douglass, William A.: "Rural Exodus in Two Spanish Basque Villages: A Cultural Explanation”, American Anthropologist, 5, 1971, pp. 1100-1101. Sobre la emigración vasca a América (referida a 
El Oeste de los Estados Unidos brindaba a los vascos oportunidades para trabajar como pastores de ovejas. Por un lado, el trabajo de ovejero no requería un conocimiento de la lengua inglesa, ni de otras nociones culturales norteamericanas necesarias para sobrevivir en otro sector económico y que casi ningún inmigrante vasco de la época dominaba. Por otro lado, el pastoreo de ganado ovino era un oficio poco demandado por la mayoría de la población blanca autóctona debido a su dureza y escasa valoración social. Además, la abundancia de tierras públicas que se podían aprovechar libremente abría una oportunidad para prosperar de la que estos inmigrantes carecían en sus lugares de origen ${ }^{13}$. Pero hay que añadir un cuarto y significativo factor: por muy distintos que fueran los inmigrantes vascos, o eso podría parecer en un primer momento debido a las diferencias culturales, al menos eran europeos blancos, y este factor facilitó su posterior integración social en el Oeste de los Estados Unidos. Por entonces, se había forjado una cadena migratoria que encauzaba el reclutamiento de la mano de obra vasca en las explotaciones ganaderas del Oeste ${ }^{14}$.

Los vascos no fueron los únicos inmigrantes de este nuevo "proletariado agrícola", expresión utilizada por Carey McWilliams en los años treinta para referirse a la mano de obra agrícola en California ${ }^{15}$. Junto a ellos también otros grupos de inmigrantes de origen no anglosajón, principalmente portugueses y mejicanos, trabajaron como pastores de ovejas en el Oeste americano ${ }^{16}$. A comienzos del siglo XX, sin embargo, se había consolidado ya en el Oeste la imagen de los vascos como mano de obra aparentemente dócil y cualificada, y que había sabido adaptarse a las duras condiciones laborales del sector, trabajando las 24 horas del día en una zona árida como la Gran Cuenca; la imagen estereotipada del best sheepherder. En 1901, en un periódico local de Iowa, se podía leer lo siguiente:

The best sheepherder in the world is the Basque, [...]. Next to him is the Mexican. Both can stand following the sheep for years, but both will succumb in time. An American hardly stands the strain for a year and I have known so me of them who did not stand it that long ${ }^{17}$.

Los inmigrantes vascos gozaron de muy buena reputación como pastores frente a otras colectividades, lo que facilitó su contratación. No obstante, en numerosas ocasiones, los grandes ganaderos del Oeste, muchos de los cuales eran también vascos que habían emigrado algunos años antes y se habían convertido en grandes produc-

una etapa anterior) véase Pildain Salazar, María Pilar: Ir a América. La emigración vasca a América (Guipúzcoa 1840-1870), San Sebastián, Caja de Ahorros Municipal de San Sebastián, 1984. Sobre la situación de crisis y guerra que vivió el País Vasco en los dos primeros tercios del siglo XIX y que impulsaron la emigración vasca a América véase Rubio Pobes, Coro, Revolución y tradición: el País Vasco ante la Revolución liberal y la construcción del Estado español, (1808-1868), Madrid, Siglo XXI, 1996.

13 Douglass, William A.: "The Basques of the American West: Preliminary Historical Perspectives", Nevada Historical Society Quarterly, 4, 1970, pp. 15-21.

14 Saitua, Iker: "Becoming Herders: Basque Immigration, Labor, and Settlement in Nevada, 1880-1910", Montana: The Magazine of Western History, 4, 2016, pp. 69-70.

15 McWilliams, Carey: Factories in the Field: The Story of Migratory Farm Labor in California, Boston, Little, Brown and Co., 1939, p. 48.

16 Véase: PEG, Arnold: "Wyoming’s Hispanic Sheepherders", Annals of Wyoming, 69, 1997, pp. 29-42; Warrin, Donald: "An Immigrant Path to Social Mobility: Portuguese Atlantic Islanders in the California Sheep Industry", California History, 4, 1997/1998, pp. 94-107; Kent Hicks, J. y Johnson, Eileen, "Pastores Presence on the Southern High Plains of Texas", Historical Archaeology, 4, 2000, pp. 46-60.

17 Des Moines Daily News, 14-XII-1901, p. 4. 
tores de ganado ovino, se aprovechaban de la fuerza de trabajo inmigrante vasca ${ }^{18}$. Era una mano de obra indefensa, al igual que otros extranjeros que trabajaban asalariados en otros sectores primarios en aquellos estados del Oeste que estaban, como ha escrito el historiador Richard White, lejos de los centros de la economía nacional, en las zonas mas remotas, aisladas y escabrosas ${ }^{19}$.

Debido a las duras condiciones de trabajo en el hostil entorno natural de Nevada, los pastores a veces morían a causa de tormentas y otras contingencias meteorológicas y ambientales. Un ejemplo documentado es el caso de Martin Goyeneche, un pastor vasco que en abril de 1915 se perdió en medio de una gran tormenta en Battle Creek, cerca de Battle Mountain (Nevada), y finalmente murió, dejando solo a todo el rebaño de ovejas que se le había encomendado ${ }^{20}$. Además de estar expuestos a tener accidentes y contraer enfermedades en las desoladas praderas de Nevada, estos pastores sufrían de una constante soledad extrema y a menudo estaban al borde de la locura. Es el caso de José Amutxastegi, quien en 1924, después de toda una vida trabajando como pastor en el desierto frío del noreste de Nevada, a sus 40 años de edad fue internado en un centro psiquiátrico por mostrar síntomas de trastorno mental $^{21}$. El trabajo de estos inmigrantes distaba mucho por consiguiente de la imagen idílica y bucólica creada en torno a la vida de los pastores vascos en las praderas del Oeste, que ha sido en cierta medida perpetuada por la historiografía. Aún más si observamos los conflictos en que se vieron inmersos por el aprovechamiento de las tierras públicas.

La creciente presencia de inmigrantes vascos en las praderas agravó el problema apremiante de la gestión y aprovechamiento de las tierras públicas y reforzó el perfil "extranjero" de este conflicto ${ }^{22}$. Grandes y medianos ganaderos que poseían propiedades dedicadas a pastos y forrajes culparon a los inmigrantes vascos del alarmante deterioro de las praderas a finales del siglo XIX, a quienes denominaban despectivamente pastores transeúntes y vagabundos. En 1899, en la renombrada revista mensual agrícola California Cultivator and Livestock and Dairy Journal, se podía leer lo siguiente:

...the sheepmen of the Sierra Nevada are for the most part a lot of irresponsible Basques, who own no other property than their sheep; they pay no taxes, evading them by moving ${ }^{23}$.

Invocando el patriotismo norteamericano de la época, los ganaderos desviaron sus ataques hacia los pastores nómadas inmigrantes para así reclamar mayor protección legal sobre el uso y manejo de las tierras públicas de Nevada y afianzar su posición económica frente a otras explotaciones ganaderas más pequeñas. Para llamar la atención del gobierno federal acerca de los problemas de tenencia de tierra y de las pugnas por el uso de aguas para abrevaderos, los ganaderos de Nevada llevaron a

18 Douglass y Bilbao: Amerikanuak..., p. 233; Entrevista, Pedro "Pete" Barinaga, August 20, 1975, transcripción, Archivo de Historia Oral del Basque Museum \& Cultural Center, Boise, Idaho; Saitua: "Becoming Herders"..., p. 69. p. 2; r Misfo.

19 White: It's Your Misfortune..., p. 270.

20 Reno Evening Gazette, 5-V-1915, p. 3.

21 Nevada State Journal, 27-VIII-1924, p. 2.

22 Hays: Conservation..., p. 54.

23 California Cultivator and Livestock and Dairy Journal, 13, 1899, p. 97. 
cabo una campaña de lucha contra los pastores vascos, que marcaría la agenda socioeconómica del Oeste árido en el primer tercio del siglo XX y que fue vivida como una auténtica persecución. Estableció además el marco interpretativo desde el que la comunidad ganadera de Nevada concibió las consecutivas crisis en la industria ganadera durante estos años ${ }^{24}$.

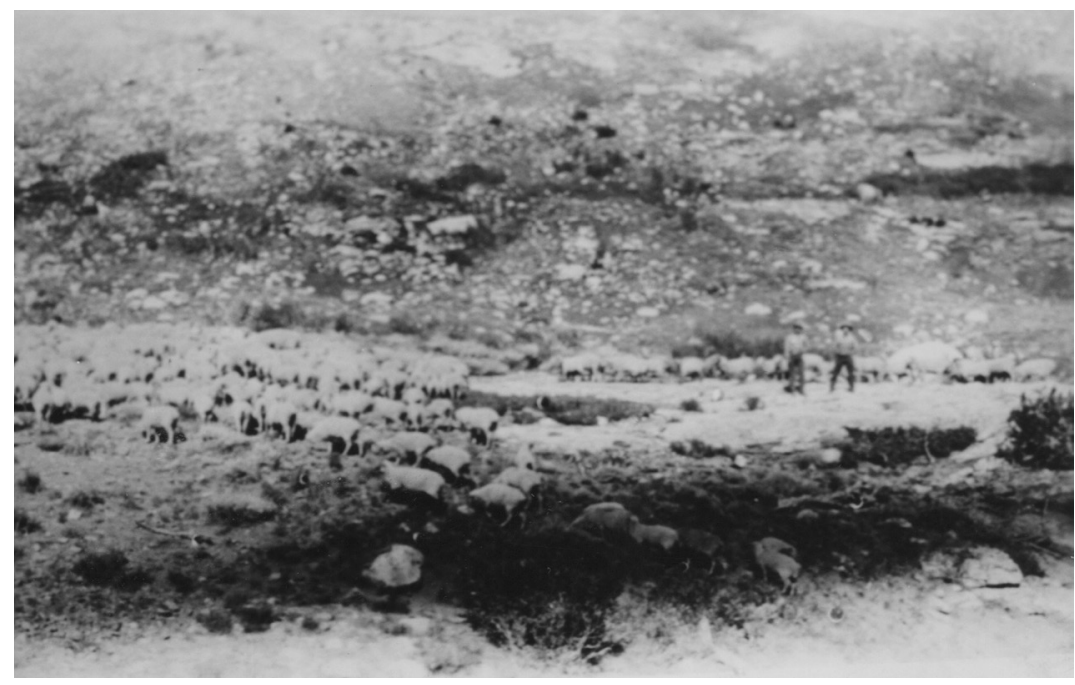

Figura 1. Dos pastores vascos sin identificar junto a su rebaño de ovejas en el noroeste de Nevada en los años 20. Fuente: número de referencia 1641-64, Northeastern Nevada Museum, Elko, Nevada.

La campaña contra los pastores vascos se convirtió también en un arma para el nuevo movimiento conservacionista, que ejerció gran influencia sobre los llamados gobiernos progresistas y sobre amplios sectores de la sociedad estadounidense a lo largo del siglo XX, y que continúa hasta nuestros días ${ }^{25}$. En 1894, John Muir, renombrado naturalista y ferviente defensor de la necesidad de preservar el medio ambiente en los Estados Unidos, escribió que las montañas de California y sus zonas colindantes con el estado de Nevada estaban siendo devastadas principalmente por el pastoreo de ovejas:

...the arch destroyers are the shepherds, with their flocks of hoofed locusts, sweeping over the ground like a fire, and trampling down every rod that escapes the plow as completely as if the whole plain were a cottage garden-plot without a fence ${ }^{26}$.

24 “Speech of Hon. Key Pittman,” Cong. Rec., 63rd Cong., 1st Sess., (August 21, 1913), Key Pittman Papers, Nevada Historical Society, Reno, Nevada; Lane, Richard H.: The Cultural Ecology of Sheep Nomadism: Northeastern Nevada, 1870-1972, tesis doctoral, University of Yale, 1974, pp. 131-137.

25 Rowley, William D.: U.S. Forest Service Grazing and Rangelands: A History, College Station, Texas A\&M University Press, 1985, pp. 15-21.

26 Muir, John: The Mountains of California, New York, The Century Co., 1907, pp. 349-350. 
El oficio de pastor de ovejas y todo lo relacionado con el sector ovino era visto como algo antiamericano, foráneo, expoliador y hostil entre la población blanca de origen predominantemente anglosajón. La figura del ovejero, que se correspondía con la del pastor vasco, parecía estar a la sombra de la imagen mítica y romantizada del vaquero o cowboy, el símbolo por antonomasia de la llamada libertad de la "frontera" americana. La influencia de la literatura popular del Oeste, divulgando esa imagen romántica del cowboy, fue decisiva en la formación de toda una generación de estadounidenses y en la vida socio-política norteamericana durante la Era Progresista ${ }^{27}$.

\section{La regularización del uso de la tierra de pastoreo en Nevada. El nuevo régimen de aprovechamiento forestal y el sector ganadero de Nevada}

Al final de la década de 1890 el gobierno federal tomó las primeras medidas para regular, gestionar y controlar la ganadería en el dominio público del Oeste. Sus áreas de pasto incluían también los suelos de los bosques naturales y otras zonas arboladas más fértiles. El pastoreo, como afirma Samuel Hays, se había convertido en la principal actividad económica en la explotación de los recursos forestales, más importante incluso que el sector maderero. Pero para comienzos del siglo XX, el pastoreo excesivo del ganado en los bosques del Oeste norteamericano era un grave problema que necesitaba de manera urgente una solución. Fue entonces cuando una elite, preocupada por la situación medioambiental, comenzó a defender una adecuada conservación y manejo de los recursos naturales. Estos conservacionistas eran en su mayoría científicos y académicos formados en las universidades prestigiosas de la costa Este, y entre ellos cabe destacar a Gifford Pinchot (1865-1946), pionero científico forestal en Estados Unidos, considerado uno de los padres de dicho movimiento. Pinchot se graduó en la Universidad de Yale y posteriormente se trasladó a Francia y Alemania para aprender ecología forestal. Su experiencia en Europa contribuyó al desarrollo de ideas novedosas relacionadas con la explotación de los bosques y demás recursos naturales en Estados Unidos, que estaban siendo devastados a causa de la industrialización y el impresionante desarrollo capitalista de finales del siglo XIX. Su aportación, junto con la incorporación de la ciencia forestal a las universidades norteamericanas y a los círculos académicos progresistas, forjarían las bases para la creación de una organización gubernamental que estableciese una explotación racional de los bosques, el Servicio Forestal ${ }^{28}$.

El 3 de marzo de 1891 el Congreso aprobó una ley que daba plena autoridad al presidente de los Estados Unidos para establecer reservas forestales: daba comienzo así a

27 Webb, Walter Prescott: The Great Plains, Lincoln, University of Nebraska Press, 1931, pp. 244-251; Rowley: "From Open Range"..., pp. 112-113. Esta generación estuvo fuertemente influida por la literatura de la época sobre estas luchas en las tierras públicas del Oeste. Numerosas novelas han abordado este episodio de la historia del Oeste. Mención especial merece la obra de Wister, Owen: The Virginian: A Horseman of the Plains, New York, Macmillan Company, 1902. Nuevas interpretaciones sobre la historia de los cowboys en el Oeste son por ejemplo: Igler, David: Industrial Cowboys: Miller \& Lux and the Transformation of the Far West, 1850-1920, Berkeley, University of California Press, 2001; Moore, Jacqueline M.: Cow Boys and Cattle Men: Class and Masculinities on the Texas Frontier, 1865-1900, New York, New York University Press, 2010.

28 Hays: Conservation..., pp. 27-32, 49. Sobre Gifford Pinchot en este periodo, véase también: Miller, Char: Gifford Pinchot: The Evolution of an American Conservationist, Milford, Grey Towers Press, 1992; Miller, Char: Gifford Pinchot and the Making of Modern Environmentalism, Washington, D.C., Island Press/Shearwater Books, 2001. 
una nueva era en la historia de la administración estadounidense de las tierras públicas que se prolongaría hasta 1964, era caracterizada por la retención y gestión de la tierra según el concepto de conservacionismo utilitario defendido por Pinchot. El 4 de junio de 1897, una nueva ley otorgó al Secretario de Interior plenos poderes para regular el manejo de las reservas forestales. Esta ley autorizaba al Departamento de Interior a regular el uso y ocupación del suelo de dichas reservas con el propósito de proteger las fuentes de agua y asegurar un suministro estable de madera que satisficiera el bien común. Aunque la ley no explicitaba la ganadería como una actividad económica permitida en estas reservas, y a pesar de ser algo confuso su contenido, como bien explicó el historiador Paul Wallace Gates, sirvió de base legal para que el gobierno controlara inmediatamente y arrendara estas zonas de los bosques dedicadas al pastoreo de ganado $^{29}$. Así, el Departamento de Interior comenzó a emitir permisos para que todo tipo de ganado - también el ovino, a pesar de la fuerte oposición de los ganaderos de vacuno- pudiese pastar de manera preferente en ellas. En el año 1902, se estableció el siguiente orden de preferencia sobre el uso de la tierra: primero, el ganado de los productores residentes en estas tierras protegidas; segundo, los animales de los ganaderos que no residiesen en estas reservas, pero que fueran propietarios de parcelas dentro de estas zonas; tercero, las cabañas de las fincas colindantes que fuesen titulares de explotaciones agropecuarias; y cuarto, el ganado de forasteros que hubiesen reclamado algunos terrenos de manera equitativa. Este sistema de explotación de la tierra persistirá tiempo después a través del programa de pastoreo de la futura agencia gubernamental Forest Service. Desde 1898 hasta 1905, Pinchot trabajó sin descanso para transferir las obligaciones del General Land Office (GLO, una oficina independiente responsable de las tierras públicas creada en 1812) al Departamento de Agricultura, ya que en su opinión los ingenieros forestales que la componían conocían mejor los bosques y demás rincones del Oeste que los juristas del Departamento de Interior. Lo consiguió: los poderes sobre las reservas forestales fueron traspasados al Departamento de Agricultura a través de la Transfer Act de $1905^{30}$.

El Servicio Forestal, capitaneado por Pinchot, fue pionero en la administración de las tierras federales durante el siglo XX. El modelo conservacionista no solo fue un ejemplo para otros organismos de protección del medio ambiente creados posteriormente en los Estados Unidos, sino que fue exportado a otras partes del mundo ${ }^{31}$. Parece que, también en este ámbito, el siglo XX puede ser considerado el siglo americano. Esta agencia gubernamental estadounidense establecía que todas aquellas tierras con recursos forestales de valor comercial, para efectos de conservación del ecosistema, debían ser protegidas, y así se catalogaron como bosques nacionales. Desde su creación, la actividad pastoril fue un asunto de gran importancia para esta agencia. El mismo Pinchot reconoció que el pastoreo de ganado era la principal actividad en los bosques nacionales a comienzos del siglo XX y por ello consideró de vital importancia la puesta en práctica de un programa para la supervivencia y fortalecimiento de la gestión silvícola, así como el establecimiento de requisitos técnicos medioambientales para afianzar la cosecha de madera y para proteger los acuíferos

29 Gates, Paul W. y Swenson, Robert W.: History of Public Land Law Development, Washington, D.C., Government Printing Office for Public Land Law Review Commission, 1968, p. 569.

30 Steen, Harold K.: The U.S. Forest Service: A History, Seattle, University of Washington Press, 1976, pp. 26-37, 69-77.

31 Ramos Gorostiza, José Luis: "Un precedente lejano del debate sobre la sostenibilidad: el Movimiento Conservacionista Americano (1890-1920)”, Información Comercial Española, ICE, 800, 2002, p. 44. 
en estas zonas de gran valor para la explotación pecuaria. Para poder velar por el uso racional de los recursos forestales, el Servicio Forestal preparó un nuevo reglamento -Use Book (1905) - en el que estableció una temporada de pastoreo y el número de cabezas pecuarias permitido. Pero fue más allá señalando que el Servicio Forestal tenía como objetivo proteger las áreas de pastizal, contribuir a mejorar la eficiencia de la industria ganadera del país y amparar a los colonos agricultores de prácticas injustas en el uso de las tierras para ganado ${ }^{32}$.

Con el fin de proteger a los ganaderos establecidos en los terrenos que ahora eran catalogados como bosques nacionales, contribuir a mejorar las áreas de pastizal y la eficiencia de la industria ganadera, el Forest Service estableció un nuevo sistema de permisos para ganaderos que distinguía tres clases de usuarios: en primer lugar, el permiso de la clase A destinado a aquellos que poseían títulos de propiedad y que durante años habían operado en estas tierras que ahora eran zonas protegidas por el gobierno central; en segundo lugar, el permiso de clase B para ganaderos que tenían título de propiedad o ranchos en las áreas contiguas a los bosques y que anteriormente habían tenido ocasión de dar de pastar al ganado en las reservas; y en tercer lugar, el permiso de la clase $\mathrm{C}$ para los itinerantes que llevaban a pastar los animales, principalmente ovejas, a las tierras públicas y no contaban con título de propiedad. Esta clasificación de permisos para pasto componía también el orden por el que el gobierno federal los repartía a los ganaderos, de manera que en caso de que las dos primeras categorías completasen la capacidad de carga de los pastos, los terceros -pastores de ovino mayoritariamente- quedaban totalmente excluidos. La administración de Theodore Roosevelt creía que debía dar preferencia a los pequeños propietarios sobre los transeúntes, categoría ésta en la que entraban los pastores vascos, a quienes perjudicó enormemente la iniciativa del Forest Service. La hostilidad de los agentes del gobierno hacia la ganadería itinerante era clara. El sistema de preferencias, que trataba de prevenir el daño que los rebaños de ovejas de los no residentes podían causar en los suelos de los bosques nacionales, sería posteriormente generalizado a otras áreas naturales administradas por otras ramas del gobierno.

En un principio, los ganaderos que utilizaban esas tierras forestales (tanto las administradas por el Servicio Forestal, dentro del Departamento de Agricultura, como las que estaban bajo el control del Departamento de Interior) lo hacían sin tener que pagar una cuota. No obstante, todos sabían que esta situación pronto iba a cambiar. Para sostener el nuevo sistema de regulación de las praderas, el Servicio Forestal estadounidense necesitaba incrementar sus ingresos y con tal fin comenzó a imponer gravámenes sobre la tierra y sobre las cabezas de ganado. Dicho brevemente, la burocratizada gestión de los recursos naturales inevitablemente significaba un aumento del gasto público y este recayó sobre los que explotaban estas tierras, entre los que estaban los ganaderos y las compañías madereras. En 1906, el Servicio Forestal introdujo un régimen de tarifas de pastoreo, por el cual todo ganadero que quisiese pastar en los bosques nacionales debía pagar una cuota proporcional al número de cabezas y tipo de ganado manejado. Si bien para algunos la regulación de las praderas significaba una mayor estabilidad y seguridad en las tierras públicas, para otros no era más que un intento de estrangular a las pequeñas explotaciones ganaderas, limitando su acceso a las tierras más ricas. Pero una mayoría criticaba la introducción

32 Steen: The U.S. Forest Service..., pp. 65-68, 78-81. 
de las tarifas por animales porque entendían que no debían pagar por este servicio del gobierno de regulación de tierras forestales. Esta disconformidad desencadenó un conflicto sociopolítico a diferentes niveles que perduró durante todo el siglo XX y que sigue siendo tema de controversia hoy día ${ }^{33}$.

El 25 de agosto de 1907, el Washington Post publicó un artículo en el que se analizaba detenidamente el papel del gobierno central y la legislación forestal para la protección del medio ambiente. En él se afirmaba que las novedades introducidas significaban acabar con la ganadería transeúnte o "inmigrante" de ganado ovino que, según reconocía, en su mayoría eran de propiedad extranjera, principalmente vasca. A pesar de haber transcurrido poco tiempo desde la creación de la nueva administración forestal, el artículo analizaba la crisis de la trashumancia ovina en las montañas de la Sierra Nevada producida como consecuencia de las medidas de regulación del uso de recursos forestales. Describía de la siguiente manera la situación generada por la nueva legislación en la región ganadera situada en el estado de California y limítrofe con Nevada:

Some of the owners of the old wandering bands have gone back to their native countries, some have entered other business, some have bought ranches and are taking even chances with the American stockmen and have become good citizens of the United States ${ }^{34}$.

La progresiva disminución de la ganadería trashumante fue la primera consecuencia de la aplicación del nuevo régimen forestal en el Oeste norteamericano. Cuando el gobierno federal comenzó a regular las tierras forestales y los precios de los pastos se incrementaron, muchos vascos que habían trabajado como ovejeros tuvieron que reciclarse o marcharse, volver a su país, cambiar de trabajo... El artículo del Washington Post ponía por otra parte de manifiesto la existencia de un interés en la opinión pública estadounidense por este tema de las tierras públicas: una ciudadanía mejor informada comenzó a comprender la importancia que el vasto dominio público tenía para el futuro del país ${ }^{35}$.

\section{El lobby ganadero de Nevada, el problema de las tierras públicas y los pastores vascos}

En lo que se refiere al estado de Nevada, la situación era algo peculiar, además de problemática. Gran parte del dominio público de este estado, como se ha señalado anteriormente, eran terrenos marginales, siendo la superficie forestal sólo una pequeña porción de este vasto territorio. Cuando el Servicio Forestal comenzó a administrar, ordenar y conservar los bosques de Estados Unidos, la mayoría de la comunidad ganadera de Nevada se mostró entusiasmada y aplaudió las medidas gubernamentales para proteger los recursos forestales, contenta porque el régimen de administración de los bosques nacionales excluía a los pastores vagabundos. Una serie de grandes ganaderos con propiedades de tierra vieron en el nuevo régimen forestal una forma

\footnotetext{
Rowley: U.S. Forest Service..., pp. 58-68.

Washington Post, 25-VIII-1907, p. 8.

Ibid.
} 
de reforzar su posición económica en el aprovechamiento de las tierras federales y de esta manera asegurarse los beneficios provenientes de las mismas. Pero pronto estos ganaderos vieron truncadas sus aspiraciones, ya que el área protegida por el Servicio Forestal en Nevada ocupaba solo una pequeña parte de todo este territorio árido y fuera de él todavía existía un vasto espacio rural donde los pastores podían operar libremente, de manera que la creación de bosques nacionales no significaba el fin para la ganadería trashumante ovina en este estado ${ }^{36}$.

De manera oportunista, los ganaderos de Nevada, agrupados en varias asociaciones locales y nacionales, comenzaron a reclamar al gobierno que ampliase estas reservas forestales para eliminar todas las actividades trashumantes de su estado. Pero estas peticiones fueron rechazadas en Washington, ya que el Servicio Forestal no consideraba estas tierras con potencial para la explotación forestal. Los rancheros locales, que dependían de las tierras de pastizal para la prosperidad de sus cabañas vacunas, se quejaban al gobierno estatal a través de los representantes de Nevada en la capital estadounidense, quienes, en gran medida, favorecieron sus intereses. Presionaron, por ejemplo, para que no se concedieran demasiadas licencias para operar ganado en los bosques, lo que favorecía por lo general a los grandes productores ganaderos de carne de vacuno. Pero además lograron que las instituciones locales impusieran gravámenes a las cabezas de ganado ovino, gravamen que por otra parte resultaba difícil de recaudar debido al constante movimiento requerido por la cría de ovejas de manera extensiva ${ }^{37}$.

Durante la década de 1910, una creciente demanda en el mercado, tanto nacional como internacional, generó un movimiento a favor de la industria ovina. En vísperas de la Gran Guerra, una rebaja de las tarifas y el incremento de la demanda de productos ovinos conllevó un aumento de la producción de ovejas ${ }^{38}$. Si por un lado el viento de la economía sopló en la dirección favorable a la industria ovina, por otro lado políticos progresistas y autoridades de Nevada se alzaron en defensa del sector vacuno. El senador demócrata de Nevada Key Pittman fue sin duda uno de los más fervientes defensores de los intereses de los productores de ganado vacuno. Marcadamente contrario a las empresas de ganado lanar, consideraba que el sector ovino suponía solamente una pequeña proporción de la riqueza estatal y que además no era una industria autóctona, porque la mayoría de empresas ganaderas de ovinos establecidas en Nevada provenían de fuera, de los estados de California, Idaho o Utah, que habían sabido sacar provecho de las ventajas que suponía deslocalizar sus explotaciones en un estado con gran porcentaje de tierras federales como Nevada. Pittman, como nacionalista y nativista acérrimo que era, colocó en el centro de sus críticas a los inmigrantes vascos empleados por la gran mayoría de las explotaciones ovinas, denunciando que, entre otras cosas, no contribuían en nada a las arcas públicas y en general a la prosperidad del estado. Decía que no eran más que mano de obra barata que fue aprovechada por un grupo importante de productores de ovinos:

36 Adams, Romanzo: "Public Range Lands: A New Policy Needed”, American Journal of Sociology, 3, 1916, pp. 324-351; Adams, Romanzo Colfax: Taxation in Nevada: a history, Carson City, State Printing Office, 1918, pp. $107,145-152$.

37 Carta de Jay H. Clemons al Servicio Forestal de los Estados Unidos (20 de marzo de 1918), Library of Congress, Key Pittman Collection (Collections of the Manuscript Division), 64, Forest Service; Adams: Taxation in Nevada..., p. 153.

38 Elliott, Russell R. (with the assistance of William D. Rowley): History of Nevada, Lincoln, University of Nebraska Press, 1973, p. 252; New York Times, 10-XII-1916, p. 8. 
The Basques come from the Pyrenees mountains in Spain. They get that class of labor because they seem to be adapted for sheep herding, and they are lacking in intelligence, independence, or anything else. They are just about as near a slave as anybody could be under our present existing conditions, $\ldots{ }^{39}$.

Pittman, en su vehemente ataque contra los pastores vascos, incluso fue más lejos e insultó a toda la colectividad vasca asentada en Nevada con argumentos como estos:

As a general thing they [Basques] never associate with other people in the state; they live among themselves; they can only speak a few words of the English language; they live in the lowest possible way for a human being to live; and they are nothing but sheep herders ${ }^{40}$.

Los ganaderos de Nevada encontraron en el senador Pittman un fuerte aliado para luchar contra las explotaciones trashumantes. El 25 de marzo de 1914, la Nevada Cattle Owners' Association le escribió una carta pidiendo especial atención al caso particular de este estado en relación a las tierras federales y los problemas derivados de su uso. Con el argumento de que existían pocas zonas de pasto productivas en $\mathrm{Ne}$ vada, lo que hacía que la cría de ganado vacuno resultara más costosa, exigieron una política agraria adecuada a las condiciones de su estado ${ }^{41}$. Por entonces, el Congreso estaba discutiendo ya varios proyectos legislativos sobre el arrendamiento de las tierras del gobierno del Oeste. El estallido de la Primera Guerra Mundial supuso un gran incremento de la demanda de productos ganaderos, tanto bovinos como ovinos. En el contexto de guerra, el Servicio Forestal flexibilizó su política de protección de bosques nacionales de forma que los ganaderos de toda clase pudiesen responder a las demandas de carne y lana. Las tensiones en las praderas se disiparon ya que, por lo general, todo el mundo sacó el máximo provecho de la coyuntura de la Gran Guerra $^{42}$. No obstante, las asociaciones de ganaderos bovinos de Nevada siguieron reclamando una mayor intervención gubernamental en la administración de las tierras públicas dedicadas al pastoreo. E1 23 de abril de 1917 Thomas Jefferson Bell, secretario del State Livestock Commission de Nevada, envió una carta a Key Pittman para que siguiese buscando soluciones para regular las actividades agropecuarias sobre las tierras federales, aun reconociendo que en esos momentos el foco de atención del gobierno federal y del pueblo norteamericano en general estaba en otros temas relevantes:

The present forest system is good but it only covers such a small part of the grazing domain. [...] All Stock Associations are in favor of Government control in the ranges. If the ranges were under control they would carry much more stock and protect the ranges from destruction ${ }^{43}$.

39 Reno Evening Gazette, 18-VI-1913, p. 4.

40 Ibid.

41 Carta de D. Staunton al Senador Key Pittman (25 de marzo de 1914), Library of Congress, Key Pittman Collection (Collections of the Manuscript Division), 64, Forest Service.

42 Dubuque Telegraph Herald, 7-IX-1916, p. 2; Rowley: U.S. Forest Service..., pp. 112-114.

43 Carta de T. J. Bell al Senador Key Pittman (23 de abril de 1917), Library of Congress, Key Pittman Collection (Collections of the Manuscript Division), 124, Correspondence, File B misc. 
En marzo de 1918, los grandes ganaderos de Nevada enviaron un informe al Departamento de Agricultura de los Estados Unidos que analizaba la frágil situación de las praderas de Nevada. El informe consideraba de vital importancia una redistribución de la tierra, criticaba todas las medidas federales adoptadas hasta el momento para regular las actividades en las tierras públicas y las tildaba de auténtico fracaso. Este documento proponía al gobierno federal una "apropiada distribución" de la tierra para el caso excepcional de Nevada, dando especial prioridad a las explotaciones agropecuarias más importantes que tuviesen tierras de heno suficientemente grandes como para cultivar una tonelada de alimento por cada cabeza de ganado y sus tierras para pasto estuviesen inmediatamente colindando con tierras de cultivo. El informe sostenía que los grandes propietarios de tierras de cultivo eran los que realmente contribuían al desarrollo económico de Nevada a través de los impuestos que pagaban al Estado, y por consiguiente también al esfuerzo bélico estadounidense en la Primera Guerra Mundial. La unión de criadores de ganado vacuno de Nevada reclamaba de esta forma apoyo gubernamental urgente para mantener su productividad y proteger la calidad nutritiva del forraje en las praderas:

We, who are one of the largest producers of livestock in Nevada respectfully ask your department to assist us during this time when help is so necessary. Give us, as well as all other producers of livestock in Nevada, the opportunity to increase production.

Call the attention of the various heads of our Government, who are at this time endeavoring to increase production, to the deplorable condition of our range lands and its future. Direct their attention to the fact that we must have help if we are going to continue to produce ${ }^{44}$.

Esta aristocracia ganadera trataba de defender sus intereses económicos frente a otras explotaciones agropecuarias estantes más pequeñas y para ello no dudó en sumarse a la lucha contra los pastores trashumantes con el fin de desviar la atención sobre el verdadero trasfondo de sus reclamaciones.

Al terminar la Primera Guerra Mundial los precios del ganado se desplomaron. Con la caída de demanda de materias primas, los bancos dejaron de conceder créditos a los ganaderos y muchas empresas pecuarias tuvieron que cerrar. A esto hay que sumar la inestabilidad que persistía en las praderas de Nevada que, como ya hemos señalado, en un elevado porcentaje eran del dominio público. Además, los ganaderos siguieron reclamando la ampliación de los bosques nacionales para excluir a los transeúntes. Tras la Gran Guerra, las relaciones entre la comunidad ganadera de Nevada y el gobierno federal se tensaron como consecuencia de una subida importante de los impuestos por el uso de las tierras para pasto, la cuota de pastoreo, una tensión que persistió durante la primera mitad del siglo XX. Según los ganaderos, el Forest Service y su sistema de cuotas de pastoreo no respondía a las necesidades locales de cada estado sino a la economía de mercado ${ }^{45}$.

44 Carta de la Union Land and Cattle Co. al Servicio Forestal, Departamento de Agricultura (20 de marzo de 1918), Library of Congress, Key Pittman Collection (Collections of the Manuscript Division), 64, Forest Service.

45 Elliott: History of Nevada..., pp. 262-265; Carta de Key Pittman a James Dysart (2 de junio de 1923), Library of Congress, Key Pittman Collection (Collections of the Manuscript Division), 104, File: Grazing Reserves; Rowley: U.S. Forest Service..., pp. 115-120. 
En marzo de 1920, los ganaderos, preocupados por la subida de impuestos, escribieron a Charles Henderson, senador de Nevada, quejándose de que el Servicio Forestal cobraba más de lo que debía en su estado, que había recaudado en el año 1917 más de lo necesario para cubrir los gastos sobre las actividades pastoriles en los bosques nacionales y que no podía aplicar esa subida de impuestos por el uso de las tierras para pasto con un criterio homogéneo en todos los estados: "Nevada should not have to stand any increase fees to make up for deficits in other parts of the country"46. En 1925, Chester Woodward, presidente de la asociación de productores de ganado vacuno de Mountain City (Nevada), comparó la administración del Servicio Forestal sobre las actividades pastoriles con un régimen feudal, donde los ganaderos eran los siervos y los agentes federales los poderosos señores que controlaban aquella sociedad ${ }^{47}$.

La crisis en la ganadería no solo afectó a la economía de Nevada, sino también repercutió sobre la población vasca de este estado, que todavía era sistemáticamente vinculada con la piratería en la industria ovina. El 7 de abril de 1924 la Quinn Canyon Livestock Association de Nevada envió una carta al senador Key Pittman explicando la crítica situación en las praderas del condado de White Pine generada, según sostenía, a causa de las actividades de los tramp sheepherders, de los "pastores vagabundos":

The spring, fall and winter ranges are not properly taken care of or controlled and as a result these three seasonal ranges are fast becoming denuded and unless some immediate action is taken to place these ranges under some form of Government control the livestock industry of the State will be completely ruined by over-grazing. The over-grazed condition on our now open public ranges is being brought about principally by the so-called "tramp-sheepmen" who drive their flocks into Nevada early in the fall and allow them to remain here until the following spring. The tramp or transient sheepman has absolutely no regard for the welfare of our ranges, his only interest being to avail himself of the maximum results and benefits of our ranges so long as they last ${ }^{48}$.

Los vascos no pudieron escapar de los sentimientos anti-inmigratorios de la época. En el año 1924, el Congreso de los Estados Unidos aprobó una ley de restricción de la inmigración con la que trataba de reducir los flujos migratorios de personas provenientes, entre otros lugares, del sur y este de Europa. Esta legislación estipulaba un sistema de cuotas basadas en orígenes nacionales que restringía el número de inmigrantes, siguiendo criterios étnicos y raciales marcadamente excluyentes. Esta ley limitó seriamente la llegada de inmigrantes vascos -mayormente aquellos que estaban sujetos a las cuotas aplicadas a España-, lo que dificultaría su contratación en el periodo de entreguerras ${ }^{49}$.

46 Carta al Senador Charles B. Henderson (6 de abril de 1920), Library of Congress, Key Pittman Collection (Collections of the Manuscript Division), Box 104, Correspondence, Legislation File: File Grazing Fees.

47 Nevada State Journal, 20-IX-1925, p. 1.

48 Carta de la Quinn Canyon Livestock Association al Senador Key Pittman (7 de abril de 1924), Library of Congress, Key Pittman Collection (Collections of the Manuscript Division), 108, Legislation File, File: Nevada National Forest.

49 Ngai, Mae M.: Impossible Subjects: Illegal Aliens and the Making of Modern America, Princeton, Princeton University Press, 2004, pp. 21-55; Lane, Richard H.: "Trouble in the Sweet Promised Land: Basques in Early 20th Century Northeastern Nevada”, en Douglass, William A., Etulain, Rirchard W. y Jacobsen, William H., Jr. 
Antes de que fuera aprobada dicha ley, en febrero de 1924, Arthur Brisbane, reputado editor norteamericano, escribió un artículo criticando las medidas anti-inmigración y remarcando que Estados Unidos no sería lo que era sin la labor de los inmigrantes. Para Brisbane solamente los inmigrantes blancos eran los que contribuían notablemente al progreso del país: "This country needs fifty million white immigrants, of the right kind and ought to have them". A su parecer, los vascos entraban dentro de esos supuestos buenos inmigrantes blancos, asimilados incluso a los anglosajones. Este texto, por muy extremo que pueda parecer, era signo de que para entonces los vascos representaban una colectividad bien asentada e integrada en el Oeste norteamericano, una comunidad definida más allá de su trabajo y dedicación en la ganadería trashumante ${ }^{50}$.

Para entonces, muchos vascos eran propietarios de tierras, ranchos y explotaciones agropecuarias, buena parte de las cuales eran grandes compañías ${ }^{51}$. Por ejemplo, Pete Itcaina, un inmigrante vasco natural de Aldudes (Baja Navarra) asentado en el noreste de Nevada que consiguió hacer una gran fortuna en el sector ganadero, y que para los años veinte era propietario de uno de los ranchos más importantes en el condado de Elko. En 1959, Itcaina vendió su rancho por un millón de dólares. Tras su muerte unos meses más tarde, el periódico de Utah Salt Lake Tribune lo recordó de la siguiente manera: "One of the West's most colorful characters" 52 . Así, aunque la ley de inmigración de 1924 supuso una importante fractura en la historia de la inmigración vasca en los Estados Unidos, a comienzos de 1920 se puede afirmar que la población inmigrante vasca estaba plenamente asentada y consolidada como residente permanente en estados como Nevada.

Pero si por una parte aparecen ya estos primeros ganaderos vascos enriquecidos, con una notable dignidad de su oficio y orgullosos de su origen, hay también que insistir en el hecho de que seguía a la vez existiendo esa especie de proletariado agrícola vasco en las praderas de Nevada que formaban los pastores asalariados (que por otra parte no eran solo vascos). Aunque en menor grado, la conflictividad en el campo persistía y todavía los pastores vascos resultaban un blanco perfecto para los ganaderos autóctonos. El 9 de julio de 1924 tuvo lugar un tiroteo entre un pastor vasco y Thomas E. Brackney (un destacado propietario y ganadero) en Grass Valley cerca de Austin (Nevada). En dicho enfrentamiento el pastor, que estaba contratado por la también vasca Sabal Estate and Sheep Company, resultó muerto. Al parecer, el nómada ovejero había introducido su rebaño en la propiedad de Brackney e invadido su terreno. Le costó la vida ${ }^{53}$. Significativamente, en ese mismo año, el escritor norteamericano Harry Sinclair Drago publicó su novela histórica Following the Grass, en la que narraba la llegada de los inmigrantes vascos a las tierras de Nevada y cómo estos se vieron envueltos en las disputas clásicas relacionadas con la posesión de las praderas entre los ovejeros y los productores de ganado vacuno ${ }^{54}$. No parece ser casual

(eds.): Anglo-American contributions to Basque studies: essays in honor of Jon Bilbao, Reno, Desert Research Institute on the Social Sciences, 1977, pp. 34-35.

50 Nevada State Journal, 12-II-1924, p. 4.

51 Lane: "Trouble"..., p. 39.

52 Salt Lake Tribune, 6-VI-1959, p. 20.

53 Reno Evening Gazette, 10-VII-1924, p. 3.

54 La novela contaba la historia de un pastor vasco llamado Anjel Irosabal que llegaba al estado de Nevada, donde encontraba un enorme territorio apropiado para la ganadería extensiva de ovinos. Drago describía de la siguiente 
que este renombrado novelista pusiera a un vasco como personaje principal en medio de aquel inhóspito Oeste.

A comienzos de los años 20, a la vez que la economía del país pasaba por uno de sus mejores momentos, los problemas en las praderas de Nevada persistían a causa del sobrepastoreo, con el consiguiente panorama de frustración generalizada de miles de ganaderos. Cada vez eran más los productores pecuarios que se quejaban de la creciente presencia de pastores que se aprovechaban de las tierras federales de Nevada como consecuencia del progresivo aumento de la ganadería estante. Pero el gobierno federal miraba hacia otro lado. Todas las leyes federales para la planificación del uso de la tierra, conservación de los recursos naturales y desarrollo rural habían fracasado en Nevada. Ante la falta de efectivas respuestas federales que dieran solución a los problemas de las praderas del Oeste, la asamblea legislativa de Nevada procedió a legislar sobre el tema y tomó importantes medidas para mitigar la inestabilidad en el sector ganadero. Pero al igual que el gobierno federal, también él fracasó en su intento de poner orden en este punto en la vida de las praderas ${ }^{55}$.

Está claro que a inicios del siglo XX, según hemos venido analizando, la comunidad ganadera había llegado a la conclusión de que era inevitable y necesaria una intervención gubernamental para estabilizar la situación en las praderas de $\mathrm{Ne}$ vada. En 1934, el pueblo norteamericano sometió a examen al joven gobierno de Franklin D. Roosevelt sobre las primeras medidas del New Deal. Por entonces, el país todavía seguía sumido en un fuerte estancamiento económico ${ }^{56}$. El 28 de junio de 1934, en medio de un panorama de gran incertidumbre, la nueva administración demócrata aprobó la Ley Taylor de Pastoreo (Taylor Grazing Act), que finalmente regulaba las vastas extensiones de tierras públicas dedicadas al pastoreo. Con la nueva ley se establecieron distritos de pastoreo y estos fueron gestionados por consejos creados por los mismos ganaderos a nivel local. Una mayoría de ganaderos de Nevada dieron la bienvenida a la nueva ley ${ }^{57}$. Unos años más tarde, un ecologista norteamericano, Kenneth Platt, definió a aquellos grandes criadores de ganado vacuno, que estuvieron durante años pidiendo al gobierno una ley que regulase las tierras de pastoreo, como "la voz que clama en el desierto" 58 . Su reclamación acabó siendo escuchada.

manera esas tierras públicas tan deseadas por los ganaderos en las que podían pastar libremente: "The soil was light, sandy-the very finest in the world for sheep. Bunch-grass, wild clover and a variety of salt brush were abundant. Timber was to hand, also. Nothing was wanting. Land was cheap”. Drago, Harry Sinclair: Following the Grass, New York, The Macaulay Company, 1924, p. 18.

55 Elmer Otis Wooton: The Public Domain of Nevada and Factors Affecting Its Use, Washington, D.C., U.S. Department of Agriculture, Technical Bulletin N. 301, April 1932, pp. 39-43.

56 Kennedy, David M.: Entre el miedo y la libertad. Los EE.UU.: de la Gran Depresión al fin de la segunda guerra mundial (1929-1945), Barcelona, Edhasa, 2005, pp. 229-234.

57 Foss, Phillip O.: Politics and Grass: The Administration of Grazing on the Public Domain, Seattle, University of Washington Press, 1960, pp. 58-63; Nevada State Journal, 20-VIII-1934, p. 4; Nevada State Journal, 25-IX1934, p. 7. Sobre la Ley Taylor de Pastoreo y el caso de la comunidad vasca, véase la tesis doctoral de Hatfield, Kevin D.: "We Were Not Tramp Sheepmen": Resistance and Identity in the Oregon Basque Community, Accustomed Range Rights, and The Taylor Grazing Act, 1890-1955, tesis doctoral, University of Oregon, 2003.

58 Nevada State Journal, 29-II-1940, p. 7. 


\section{Conclusiones}

En la década de 1890, el sobrepastoreo, las roturaciones desmesuradas y las sucesivas crisis ambientales habían deteriorado de manera preocupante el suelo y la vegetación para uso ganadero. En este contexto de crisis generalizada, en medio de un ambiente de extrema incertidumbre económica, el frágil equilibrio entre ganadería bovina y ovina se resquebrajó por completo a causa de la llegada masiva de nuevos inmigrantes que se apuntaron a esta empresa agropecuaria que parecía ser una fuente de trabajo segura. Al inicio del siglo XX, con la enorme difusión de las ovejas en el estado de Nevada, y otros estados del Oeste norteamericano, los inmigrantes europeos que llegaron allí, entre los que destacaban los vascos, ocuparon los puestos de pastores que el sector ovino, en una etapa de bonanza económica, demandaba. Por ese entonces, ganadería trashumante y mano de obra vasca se convirtieron rápidamente en dos caras de la misma moneda. Pero para los grandes productores de carne de vacuno, estos pastores nómadas se convirtieron en una amenaza, e igualmente para otras explotaciones ganaderas más pequeñas, que además de sus propios terrenos también utilizaban como pastos las tierras del gobierno. La falta de intervención gubernamental en el ordenamiento territorial generó un panorama de inestabilidad socio-económica en el que fueron frecuentes las luchas entre ganaderos por reservarse el control y usufructo de las tierras federales más productivas. Esto hizo necesario que el gobierno federal tomase cartas en el asunto. Pero entre 1905 y 1934, las únicas tierras públicas de Nevada administradas por el gobierno federal fueron las zonas declaradas como bosques nacionales: en Nevada estas áreas protegidas solamente comprendían una minúscula parte de todo el territorio estatal. Fuera de estos bosques nacionales, el vasto dominio público dedicado a la ganadería no estaba regulado por ninguna agencia del gobierno federal y el Servicio Forestal no estaba dispuesto a asumir la carga burocrática que significaba administrar estas tierras. Esta situación prolongó la inestabilidad en las praderas y las disputas por la utilización de las tierras públicas.

La posición de la comunidad vasca en la industria ovina del estado de Nevada se deterioró durante e inmediatamente después de la Primera Guerra Mundial, cuando los grandes ganaderos defendieron su posición económica en las tierras federales y sus derechos como ciudadanos estadounidenses. La asamblea legislativa de Nevada y entidades privadas de este estado se esforzaron por establecer un orden en las praderas que salvaguardara sus intereses, pero todas las medidas y mecanismos para excluir a los pastores nómadas fracasaron. El gobierno federal por su parte parecía tener preocupaciones mayores y no asumió un decidido papel de regulador y de árbitro. Así, la crisis de la ganadería lanar trashumante se alargó en Nevada hasta que finalmente se aprobó en 1934 la Ley Taylor de Pastoreo. Para entonces, algunos vascos se habían convertido en importantes propietarios y a pesar de que muchos seguían pastoreando sus ovejas de manera extensiva sin tener ninguna propiedad, la mayoría llevaba años combinando el uso de las tierras públicas y las parcelas privadas. Con su trabajo en las montañas, aquellos vascos habían consolidado su posición social dentro de la sociedad norteamericana y habían dejado de ser aquellos tramp sheepherders, apreciados como buena mano de obra pero blancos de las disputas ganaderas en las praderas. El largo camino recorrido hasta conseguir la ley Taylor, los reiterados intentos legislativos desde instancias estatales y federales por poner orden en las praderas, pone de manifiesto que todavía a finales de la década de 1920 no era 
una realidad lo que apuntaba aquel periódico local de 1913 al que nos referíamos al comenzar este artículo: "Range wars are ended". Los conflictos sobre las praderas de Nevada no terminaron, como se esperaba, tras la creación del Servicio Forestal sino que persistieron durante muchos años, afectando a los pastores ovejeros, y en consecuencia a la comunidad vasca de inmigrantes.

\section{Referencias bibliográficas}

Adams, Romanzo Colfax: Taxation in Nevada: a history, Carson City, State Printing Office, 1918.

_ "Public Range Lands: A New Policy Needed", American Journal of Sociology, 3, 1916, pp. 324-351.

Arrizabalaga, Marie Pierre: A Statistical Study of Basque Immigration into California, Nevada, Idaho and Wyoming Between 1900 and 1910, tesis de master, University of Nevada, Reno, 1986.

Douglas, William A. y Bilbao, Jon: Amerikanuak: Basques in the New World, Reno, University of Nevada Press, 1975.

- Etulain, Rirchard W. y Jacobsen, William H., Jr. (eds.): Anglo-American contributions to Basque studies: essays in honor of Jon Bilbao, Reno, Desert Research Institute on the Social Sciences, 1977.

— "Rural Exodus in Two Spanish Basque Villages: A Cultural Explanation", American Anthropologist, 5, 1971, pp. 1100-1114.

- "The Basques of the American West: Preliminary Historical Perspectives", Nevada Historical Society Quarterly, 4, 1970, pp. 12-25.

Drago, Harry Sinclair: Following the Grass, New York, The Macaulay Company, 1924.

Elliott, Russell R. (with the assistance of William D. Rowley): History of Nevada, Lincoln, University of Nebraska Press, 1973.

Foss, Phillip O.: Politics and Grass: The Administration of Grazing on the Public Domain, Seattle, University of Washington Press, 1960.

Gates, Paul W. y Swenson, Robert W.: History of Public Land Law Development, Washington, D.C., Government Printing Office for Public Land Law Review Commission, 1968.

Hatfield, Kevin D.: “We Were Not Tramp Sheepmen": Resistance and Identity in the Oregon Basque Community, Accustomed Range Rights, and The Taylor Grazing Act, 18901955 ", tesis doctoral, University of Oregon, 2003.

Hays, Samuel P.: Conservation and the Gospel of Efficiency: The Progressive Conservation Movement, 1890-1920, Cambridge, Harvard University Press, 1959.

Igler, David: Industrial Cowboys: Miller \& Lux and the Transformation of the Far West, 1850-1920, Berkeley, University of California Press, 2001.

Kennedy, David M.: Entre el miedo y la libertad. Los EE.UU. de la Gran Depresión al fin de la segunda guerra mundial (1929-1945), Barcelona, Edhasa, 2005.

Kent Hicks, J. y Johnson, Eileen, "Pastores Presence on the Southern High Plains of Texas", Historical Archaeology, 4, 2000, pp. 46-60.

Lane, Richard H.: The Cultural Ecology of Sheep Nomadism: Northeastern Nevada, 18701972, tesis doctoral, University of Yale, 1974.

McWilliams, Carey: Factories in the Field: The Story of Migratory Farm Labor in California, Boston, Little, Brown and Co., 1939. 
Miller, Char: Gifford Pinchot and the Making of Modern Environmentalism, Washington, D.C., Island Press/Shearwater Books, 2001.

- Gifford Pinchot: The Evolution of an American Conservationist, Milford, Grey Towers Press, 1992.

Moore, Jacqueline M.: Cow Boys and Cattle Men: Class and Masculinities on the Texas Frontier, 1865-1900, New York, New York University Press, 2010.

Muir, John: The Mountains of California, New York, The Century Co., 1907.

Ngai, Mae M.: Impossible Subjects: Illegal Aliens and the Making of Modern America, Princeton, Princeton University Press, 2004.

Patterson, Edna B.; Ulph, Louise A. y Goodwin, Victor: Nevada's Northeast Frontier, Sparks, Western Printing and Publishing Company, 1969.

Peffer, Louise E.: The Closing of the Public Domain: Disposal and Reservation Policies, 1900-1950, Stanford, Stanford University Press, 1951.

PEG, Arnold: "Wyoming's Hispanic Sheepherders", Annals of Wyoming, 69, 1997, pp. 29-42.

Pildain Salazar, María Pilar: Ir a América. La emigración vasca a América (Guipúzcoa 18401870), San Sebastián, Caja de Ahorros Municipal de San Sebastián, 1984.

Ramos Gorostiza, José Luis: "Un precedente lejano del debate sobre la sostenibilidad: el Movimiento Conservacionista Americano (1890-1920)", Información Comercial Española, ICE, 800, 2002, pp. 31-46.

Robbins, William G. y Foster, James C. (eds.): Land in the American West: Private Claims and the Common Good, Seattle-London, University of Washington Press, 2000.

Rowley, William D.: U.S. Forest Service Grazing and Rangelands: A History, College Station, Texas A\&M University Press, 1985.

Rubio Pobes, Coro, Revolución y tradición: el País Vasco ante la Revolución liberal y la construcción del Estado español, (1808-1868), Madrid, Siglo XXI, 1996.

Saitua, Iker: "Becoming Herders: Basque Immigration, Labor, and Settlement in Nevada, 1880-1910", Montana: The Magazine of Western History, 4, 2016, pp. 58-70.

Sawyer, Byrd W.: Nevada Nomads: A Story of the Sheep Industry, San Jose, Harlan-Young Press, 1971.

Steen, Harold K.: The U.S. Forest Service: A History, Seattle, University of Washington Press, 1976.

Warrin, Donald: “An Immigrant Path to Social Mobility: Portuguese Atlantic Islanders in the California Sheep Industry”, California History, 4, 1997/1998, pp. 94-107.

Webb, Walter Prescott: The Great Plains, Lincoln, University of Nebraska Press, 1931.

White, Richard: "It's Your Misfortune and None of My Own": A New History of the American West, Norman, University of Oklahoma Press, 1991.

Wister, Owen: The Virginian: A Horseman of the Plains, New York, Macmillan Company, 1902.

Young, James A. y SparkS, B. Abbott: Cattle in the Cold Desert, Logan, Utah State University Press, 1985. 